\title{
COORDENAÇÃO MOTORA E ÍNDICE DE DESENVOLVIMENTO DA EDUCAÇÃO BÁSICA: UMA RELAÇÃO PEDAGÓGICA
}

\author{
Jenipher Emanuelle de Lima Campos Zampier Correio \\ Universidade Federal de Ouro Preto, Ouro Preto, Minas Gerais, Brasil
}

Siomara Aparecida Silva

Universidade Federal de Ouro Preto, Ouro Preto, Minas Gerais, Brasil

\begin{abstract}
Resumo
O objetivo deste estudo é analisar a relação entre a coordenação motora e o Índice de Desenvolvimento da Educação Básica (Ideb), bem como se há interferência da quantidade de prática esportiva nessa relação pedagógica. Participaram 154 alunos dos Anos Iniciais e Finais do Ensino Fundamental. Realizaram-se duas coletas (pré e pós-teste) através do teste KTK, em dois grupos. Através do T-Student independente e pareado foram feitas as comparações. Verificou-se uma relação direta entre o Ideb e a coordenação, não significativa e, interferência da prática esportiva e do contexto. A relação pedagógica estabelecida reafirma a necessidade de uma prática esportiva alicerçada na capacidade cognitiva com vistas na melhoria da qualidade da ação motora e da qualidade do ensino.
\end{abstract}

Palavras-chave: Educação. Educação Física e Treinamento.

\section{Introdução}

A escola é hoje, o espaço mais privilegiado para a construção e disseminação de valores e hábitos positivos que possam perdurar
por todo o período de vida de um indivíduo (VALDÍVIA et al, 2008). Deveria-se reunir na escola, todas as crianças (adolescentes e jovens) em idade de favorecimento de aprendizagem, como previsto pela legislação. A democratização da educação no Brasil, com vistas ao acesso à escola, já é uma realidade, sendo agora, a qualidade do ensino a realidade almejada.

Recentemente, foi criado no Brasil um indicador sintético com o objetivo de monitorar a qualidade do ensino a partir do desenvolvimento escolar (FERNANDES, 2007). O Índice de Desenvolvimento da Educação Básica (Ideb) une a pontuação média dos estudantes em provas padronizadas (Prova Brasil e Sistema de avaliação da Educa- 
ção Básica) de Português e Matemática em cada etapa final da educação básica e a taxa média de aprovação dos estudantes da correspondente etapa de ensino. A utilização de um indicador como esse permite a detecção de instituições e/ou redes cujos escolares apresentem baixo desempenho e o monitoramento da evolução temporal do rendimento dos alunos.

A qualidade do ensino abrange muitos outros fatores além dos contemplados pelo Ideb, envolve todo o processo de ensino aprendizagem e o contexto social no qual o estudante está inserido. Reunir em um único indicador parâmetros que evidenciam um ensino de qualidade é algo até o momento não disponível. Uma alternativa seria estabelecer relações pedagógicas entre características mensuráveis capazes de influenciar e sofrer influência, no e do ensino aprendizado, respectivamente.

Nesse contexto, a Educação Física se mostra como aliada, quando se tem no seu processo educacional a coordenação motora. A coordenação é um componente complexo e relevante capaz de auxiliar as tomadas de decisões e nortear a elaboração de intervenções nas aulas de Educação Física (GORLA; DUARTE; MONTAGNER, 2008). Essa capacidade, a ser desenvolvida na infância, é importante pela interação harmoniosa e econômica dos sistemas sensorial, nervoso e músculo-esquelético na execução de movimentos precisos e equilibrados (KIPHARD, 1976), almejando uma economia do sistema e o alcance do objetivado.

A coordenação motora tem sua importância pedagógica, epidemiológica e psicomotora reconhecida. O nível coordenativo não só é determinante no desenvolvimento escolar como também no desenvolvimento integral do aluno, mantendo assim, uma estreita relação entre o que o indivíduo é capaz de realizar com o que é capaz de aprender (VALDÍVIA et al, 2008; NETO et al, 2010). Ainda, a coordenação motora é influenciada por indicadores sociais como o nível socioeconômico, padrão alimentar, estilo de vida, além de sofrer influência da idade, do sexo, e de fatores ambientais (VALDÍVIA et al, 2008) que configuram na composição da cultura.

Tanto o indicador de qualidade de ensino, o Ideb, quanto o nível coordenativo são capazes de diagnosticar dificuldades e potencialidades, seja no sistema de ensino ou no próprio desempenho dos estudantes. Essa detecção sendo precoce é um importante recurso para lidar com um problema generalizado no Brasil: o fracasso escolar - 
que tem repercussões para toda a vida. Além disso, reafirma a necessidade de proporcionar aos escolares uma gama de experiências motoras, tendo a ludicidade como pressuposto, visto as implicações que o alargamento motor tem no desenvolvimento intelectual e vice-versa. Chamando a atenção para a importância da qualidade da Educação Física escolar que, além do seu papel disciplinador, deve possuir sistematização e diversificação no trato com a cultura corporal de movimento, considerando a corporeidade como elemento da formação humana (MINAS GERAIS, 2007a, 2007b).

Baseado nestas estruturas, o objetivo deste estudo é analisar a relação entre a coordenação motora e o Índice de Desenvolvimento da Educação Básica, bem como se há interferência da quantidade de prática esportiva nessa relação pedagógica.

\section{Metodologia}

Após a aprovação do trabalho pelo Comitê de Ética em Pesquisa da Universidade Federal de Ouro Preto (0004.0.238.000-09), os pais e/ou responsáveis de 154 alunos assinaram o termo de consentimento livre e esclarecido autorizando que seus filhos participassem desse estudo.

A amostra de ambos os sexos estavam entre 8 a 15 anos de idade $(\mathrm{M}=11,50 \pm 2,45)$. Distribuídos em 10 escolas municipais e 3 estaduais que oferecem ensino aos Anos Iniciais, sendo 48 e 17 alunos respectivamente, totalizando 65 alunos. Outros 54 alunos estavam dispostos em 5 escolas municipais ( 25 alunos) e 4 estaduais ( 29 alunos) que oferecem ensino aos Anos Finais. Além desses, 29 alunos da etapa Anos Iniciais da rede municipal, participaram do grupo controle (GC) no primeiro momento (pré-teste) e 35 do mesmo grupo, no segundo momento (pós-teste), sendo um total de 35 participantes do GC. O grupo teste (GT) foi constituído por alunos dos Anos Iniciais. A coleta realizada em dois momentos teve um intervalo de aproximadamente seis meses no ano letivo de 2010.

Através de uma pesquisa no site oficial do Ideb foi coletado o resultado municipal e estadual desse indicador referente ao ano de 20092010 , bem como de cada uma das escolas participantes do estudo. Foram utilizados valores médios do Ideb, tendo em vista as escolas envolvidas na pesquisa. 
O instrumento utilizado para avaliar a coordenação motora foi o Körprkoordinationstest für kinder (KTK) (KIPHARD; SCHILLING, 1974) que tem confiabilidade $(r=0.90)$ estabelecida pelo método de teste/reteste. É constituído por quatro tarefas, trave de equilíbrio (EQ), saltos monopedais (SM), salto lateral (SL) e transferência entre plataformas (TP), seguiu-se o protocolo de Gorla e Araújo (2007). Ainda que duas (SM e SL) das quatro provas do KTK possuam tabelas normativas separadas por sexos, os dados obtidos nessa pesquisa não foram analisados fazendo tal separação, uma vez que o Ideb não faz esta distinção, assim, todas as tabelas foram consultadas.

O grupo controle só participou regularmente das aulas de Educação Física escolar e das atividades do cotidiano, não controladas. Os alunos do grupo teste, além de participarem regularmente das aulas de Educação Física e das atividades do cotidiano não controladas, também eram beneficiados de um programa social de caráter esportivo em três dias da semana. Este adotava uma proposta metodológica de ensino universal dos esportes (Iniciação Esportiva Universal).

A análise estatística compreendeu em medidas de tendência central, média e desvio-padrão, bem como o teste T-Student independente e pareado, visto que através do Kolmogorv-Smirnov os dados foram aceitos como normais. Utilizou-se o pacote estatístico PASW 18.

\section{Resultados}

A análise dos resultados mostrou que tanto as escolas municipais quanto as estaduais, participantes desse estudo, apresentaram Ideb superior ou igual aos seus pares (Ideb Municipal e Ideb Estadual). Com vistas ao nível de coordenação motora, obtida através do quociente motor (QM - KTK) percebeu-se diferença entre as duas redes de ensino público, porém não significativa $[\mathrm{t}(0,416), \mathrm{p}=0,681]$, adotando $\mathrm{p}<0,05$. Entretanto, pode-se verificar uma relação direta do Ideb com a coordenação motora, ou seja, maior Ideb, maior nível de coordenação motora, sendo o inverso verdadeiro. $\mathrm{O}$ descritivo dos resultados das escolas municipais e estaduais (Anos Iniciais) está apresentado na tabela 1 . 
Tabela 1: Descritivo das escolas - Anos Iniciais.

\begin{tabular}{|c|c|c|c|c|}
\hline $\begin{array}{l}\text { Escolas } \\
\text { Anos } \\
\text { Iniciais }\end{array}$ & $\begin{array}{c}\text { QM KTK } \\
\text { (Média e Desvio } \\
\text { Padrão) }\end{array}$ & Média Ideb & $\begin{array}{l}\text { Ideb Municipal } \\
\text { Anos Iniciais }\end{array}$ & $\begin{array}{l}\text { Ideb Estadual Anos } \\
\text { Iniciais }\end{array}$ \\
\hline $\begin{array}{c}\text { Municipais } \\
(\mathrm{n}=48)\end{array}$ & $86,64 \pm 2,12$ & 5,1 & 4,9 & 5,4 \\
\hline $\begin{array}{l}\text { Estaduais } \\
(\mathrm{n}=17)\end{array}$ & $88,29 \pm 3,35$ & 5,4 & & \\
\hline
\end{tabular}

Comparando o QM-KTK e o Ideb entre duas escolas municipais, localizadas em bairros com características peculiares distintas, os resultados não demonstraram diferenças significativas $[\mathrm{t}(1,256)$, $\mathrm{p}=0,221]$.

Os resultados da tabela 2 apresentam a influência socioambiental na relação Ideb e coordenação motora.

Tabela 2: Escola Municipal 1 x Escola Municipal 2 - contextos diferentes.

\begin{tabular}{ccc}
\hline & QM KTK (Média e Desvio Padrão) & Ideb \\
\hline $\begin{array}{c}\text { Escola Municipal 1 } \\
(\mathrm{n}=15)\end{array}$ & $84,06 \pm 4,46$ & 4,6 \\
$\begin{array}{c}\text { Escola Municipal 2 } \\
(\mathrm{n}=31)\end{array}$ & $90,54 \pm 2,60$ & 5,4 \\
\hline
\end{tabular}

Com relação ao nível coordenativo, entre as redes de ensino nos Anos Finais, foram encontrados valores diferentes entre as escolas municipais, porém sem significância $[\mathrm{t}(-0,707), \mathrm{p}=0,483]$, quando comparado com as escolas estaduais. Verifica-se também que as escolas participantes desse estudo apresentaram médias do Ideb superiores às médias gerais municipais e estaduais, respectivamente.

Tabela 3: Descritivo das escolas - Anos Finais.

\begin{tabular}{|c|c|c|c|c|}
\hline $\begin{array}{c}\text { Escolas } \\
\text { Anos Finais }\end{array}$ & $\begin{array}{c}\text { QM KTK (Média } \\
\text { e } \\
\text { Desvio Padrão) }\end{array}$ & Média Ideb & $\begin{array}{l}\text { Ideb Municipal } \\
\text { Anos Finais }\end{array}$ & Ideb Estadual Anos Fina is \\
\hline $\begin{array}{l}\text { Municipais } \\
(\mathrm{n}=25)\end{array}$ & $85,48 \pm 3,66$ & 4,8 & 3,5 & 4,4 \\
\hline $\begin{array}{l}\text { Estaduais } \\
(\mathrm{n}=29)\end{array}$ & $81,69 \pm 3,92$ & 4,6 & & \\
\hline
\end{tabular}

A comparação, nos Anos Iniciais entre os momentos (pré e póstestes) do GT e do GC, mostrou uma diferença significativa [t($10,410), \mathrm{p}=0,001]$ e $[\mathrm{t}(-8,715), \mathrm{p}=0,001]$, respectivamente, para nível de coordenação motora (QM-KTK). Comparando os grupos no préteste não foi verificada diferença significativa [t(-1,459), $\mathrm{p}=0,149]$, entre eles. Comparando os grupos no pós-teste também não se identificou diferenças significativas $[\mathrm{t}(1,617), \mathrm{p}=0,112]$. O teste T-Student 
pareado mostrou que na comparação entre os momentos nos Anos Finais os grupos são diferentes significativamente $[\mathrm{t}(-4,437), \mathrm{p}=0,001]$.

Tabela 4: Pré e pós-testes - Anos Iniciais e Anos Finais.

\begin{tabular}{|c|c|c|c|c|c|c|c|}
\hline & & \multirow{2}{*}{$\begin{array}{c}\text { Anos } \\
\text { Iniciais } \\
\mathrm{GC}(\mathrm{n}=35)\end{array}$} & \multirow{2}{*}{$\begin{array}{l}\text { Pré-teste } \\
\text { GT (n=65) }\end{array}$} & \multirow{2}{*}{$\begin{array}{c}\text { Pós-teste } \\
\text { GC (n=29) }\end{array}$} & \multicolumn{3}{|c|}{ Delta (pós-pré) } \\
\hline & & & & & GT $(n=65)$ & $\mathrm{GC}$ & GT \\
\hline $\begin{array}{l}\text { QM- } \\
\text { KTK }\end{array}$ & & $87,97 \pm 2,39$ & $87,08 \pm 1,78$ & $95,69 \pm 2,86^{*}$ & $101,08 \pm 1,70^{*}$ & 7,72 & 14 \\
\hline $\begin{array}{c}\text { e } \\
\text { Desvio } \\
\text { Padrão) }\end{array}$ & $\begin{array}{c}\text { Anos } \\
\text { Finais } \\
(\mathrm{n}=54)\end{array}$ & $83,44 \pm 2,69$ & $93,52 \pm 2,27 *$ & & & & \\
\hline
\end{tabular}

\section{Discussão}

Os estudos que se destinam a averiguar a participação dos componentes motores nas habilidades cognitivas enfatizam o quanto o aspecto motor é relevante no desenvolvimento global do indivíduo e ainda estabelecem a avaliação motora como uma ferramenta de diagnóstico capaz de prevenir ou sanar problemas que possam prejudicar o processo de escolarização, como também nortear ações interventivas no mesmo.

Neto et al (2011) investigaram a influência do esquema corporal de 39 escolares de 6 a 10 anos de idade com dificuldades de aprendizagem. Sugeriram que o desenvolvimento das capacidades motoras, em especial do esquema corporal, é fundamental para o desenvolvimento de habilidades essenciais à aprendizagem escolar. Amaro et al (2010) numa amostra composta por 38 escolares com dificuldades de aprendizagem, verificaram déficits motores através da Escala de Desenvolvimento Motor (EDM), concluíram que existe um forte elo entre os aspectos escolares, principalmente rendimento escolar, e o comportamento motor de crianças.

Fin e Barreto (2010) num estudo com o objetivo de verificar o perfil do desenvolvimento motor (EDM) de escolares, 60 indivíduos de 6 a 15 anos de idade, identificados como crianças, que apresentavam algum tipo de dificuldade no aprendizado escolar, constataram na análise dos dados uma relação entre as dificuldades de aprendizagem com o desenvolvimento motor, uma vez que a maior parte dos escolares apresentou atrasos motores. Os autores perceberam que os maiores déficits motores encontrados tem uma estreita relação com a capaci- 
dade coordenativa ampla (equilíbrio, esquema corporal e organização espacial).

O presente estudo ao relacionar o Índice de Desenvolvimento da Educação Básica (Anos Iniciais e Finais) com o nível coordenativo de crianças e adolescentes também demonstra a interferência da motricidade no desempenho escolar. Uma vez que os resultados mostraram que um maior nível coordenativo está relacionado com um maior Ideb.

Os estudos discutidos tem em comum a dificuldade de aprendizagem e o déficit motor, o que neste estudo não foi objeto de investigação. Nessas pesquisas utilizou-se a Escala de Desenvolvimento Motor (NETO et al, 2010) como instrumento que avalia os componentes da motricidade humana, são eles: esquema corporal, equilíbrio, organização espacial e temporal, motricidade fina e global. O instrumento KTK objetiva fornecer quociente motor da coordenação corporal através do equilíbrio, energia dinâmica das extremidades inferiores, velocidade de movimentos e velocidade combinada (GORLA; ARAÚJO, 2007).

Diante disso, nota-se que os instrumentos de medida, tanto o KTK como a EDM, tem em comum o construto coordenação motora o que justifica as comparações e a apropriação das conclusões unânimes, inerentes à relação entre desempenho motor e desempenho escolar.

Medina-Papst e Marques (2010) utilizando-se do instrumento EDM avaliaram o desenvolvimento motor em três idades, 8, 9 e 10 anos de crianças com dificuldades na aprendizagem. Constataram que todos os grupos apresentaram atrasos motores e, além disso, os resultados demonstraram que quanto maior a idade, maiores os atrasos motores.

Contribuindo com isto, Gramani e Duarte (2011) buscaram relacionar o desempenho da Educação Básica com a qualidade do Ensino Superior. Os resultados mostraram que as notas do Ideb para o Ensino Fundamental nos Anos Iniciais possuem um maior potencial de melhorias, ou seja, esta etapa da educação é a que mais exerce influência na qualidade do Ensino Superior segundo esse estudo.

$\mathrm{O}$ atual estudo corrobora com esse achado quando os resultados evidenciaram que o nível de coordenação motora influencia na nota do Ideb. A faixa etária favorável para o desenvolvimento da coordenação motora se finda no início da etapa Anos Finais do Ensino Fundamental (SILVA, 2010; GRECO; SILVA; SANTOS, 2009; 
KRÖGER; ROTH, 2002). Sendo assim, uma atenção maior dada a este componente da motricidade humana, nesta fase, certamente contribuirá com o aumento da nota do Ideb, consequentemente com a qualidade do Ensino Superior.

Nessa perspectiva uma maior diversidade de atividades oportunizadas é um pressuposto essencial para a melhoria da coordenação motora. O programa social de cunho esportivo, o qual o GT fazia parte, está pautado na proposta metodológica da Iniciação Esportiva Universal (IEU) através do método incidental para o ensino dos esportes. Nesse programa, aos escolares, eram proporcionadas experiências motoras variadas visando à sedimentação do repertório motor, ampliando assim, o seu "baú de experiências" (GRECO; SILVA, 2008). Atribui-se os resultados superiores do GT entre os momentos (pré e pós-testes) quando comparados com o GC à maior quantidade de prática que aquele grupo estava submetido, e à diversidade de atividades sustentada pela proposta metodológica.

Amaro (2010) investigou a influência de um programa de intervenção motora nos aspectos motores, acadêmicos e sociais de escolares (Anos Iniciais do Ensino Fundamental) com dificuldades na aprendizagem. A autora concluiu que as intervenções motoras influenciaram de maneira significativa na motricidade e também para além do âmbito motor, uma vez que promoveu efeitos positivos no rendimento escolar e no comportamento dos escolares que participaram do programa. Esses achados corroboram com os atuais no que tange a melhora do nível coordenativo (GT) após um tempo de prática e enfatizam sua necessidade nos Anos Iniciais.

Sob a ótica dos condicionantes da motricidade (KRÖGER; ROTH, 2002) é possível aproximar os parâmetros para o treinamento da coordenação motora das quatro provas que compõem o KTK. Na prova trave de equilíbrio objetiva-se avaliar a coordenação sob o condicionante pressão de precisão. Nas provas saltos monopedais e transferência entre plataformas sob o condicionante de pressão de complexidade. Já na prova salto lateral, o condicionante pressão de tempo (EHLING; GRECO, 2005). Estes parâmetros da coordenação motora estão contidos na proposta da IEU.

Os resultados da comparação entre duas escolas municipais sugerem uma relação direta entre o QM-KTK e o Ideb, sendo que, um maior nível de coordenação motora está relacionado a um maior Ideb. 
Embora não tenham sido coletadas informações que caracterizem, mais precisamente, o fator socioambiental dos bairros os quais essas escolas estão situadas, percebe-se que a relação sofre influência deste fator. Neto e colaboradores (2004) verificaram que o ambiente social e as práticas diárias interferem na performance motora de meninos. Valdívia et al (2008) estudaram a influência do nível socioeconômico na coordenação motora utilizando as escolas como critério (privada, pública-zona urbana e pública-zona urbana marginal). Observaram que pertencer a determinado nível influencia o desempenho motor de escolares, tanto positivamente quanto negativamente. Afirmaram ainda que, uma série de estímulos ambientais desfavoráveis provocam adaptação no comportamento motor favorecendo uma ou outra habilidade em desenvolvimento.

Assim, a relação pedagógica estabelecida entre a coordenação motora e o Índice de Desenvolvimento da Educação Básica reafirma a necessidade de uma prática esportiva alicerçada na capacidade cognitiva, a fim de melhorar a qualidade da ação motora e contribuir consequentemente com a qualidade do ensino. Portanto, uma atenção dos professores de Educação Física é requerida no sentido de se comprometerem com a qualidade das aulas e com o processo de ensino, contemplando a diversidade inerente ao ambiente escolar.

Motor Coordination and Development Index Basic Education: a pedagogical relationship

\begin{abstract}
The objective of this study is to analyze the relationship between motor coordination and the Index of Basic Education Development (Ideb) as well as for interference on the amount of sports that the pedagogical relationship. 154 students participated in the Initial and Final Years of elementary school. It was made two collections (pre-and post-test) through the test KTK into two groups. Through the Student T-independent and paired comparisons were made. There was a direct relationship between Ideb and coordination, but not significant, and interference of sports practice and context. The pedagogical relationship established reaffirms the need for a sports practice grounded in cognitive ability in order to improve the quality of motor action and the quality of education.
\end{abstract}

Keywords: Education. Physical Education and Training. 
Motor de Coordinación y Desarrollo de la Educación Índice Básico: una relación pedagógica

\section{Resumen}

El objetivo de este estudio es analizar la relación entre la coordinación motora y el Índice de Desarrollo de la Educación Básica (Ideb), así como la interferencia de la cantidad de deportes que la relación pedagógica. 154 estudiantes participaron en los años iniciales y finales de la escuela primaria. Se hizo dos colecciones (pre-y post-test) a través de la prueba de KTK, en dos grupos. A través de la T-Student comparaciones independientes y pareadas fueron hechas. Hubo una relación directa entre Ideb y la coordinación, pero no significativo, y la interferencia de la práctica deportiva y el contexto. La relación pedagógica establecida reafirma la necesidad de una práctica deportiva basada en la capacidad cognitiva con el fin de mejorar la calidad de la acción motora y la calidad de la educación.

Palabras clave: Educación. Educación y Entrenamiento Físico.

\section{Referências}

AMARO, K. N. Intervenção motora para escolares com dificuldades na aprendizagem. 2010. 57 f. Dissertação (Mestrado em Ciências do Movimento Humano) -Centro de Ciências da Saúde e do Esporte. Universidade do Estado de Santa Catarina, Florianópolis, 2010.

AMARO, K. N. et al. Desenvolvimento motor em escolares com dificuldades na aprendizagem. Movimento \& Percepção, Espírito Santo do Pinhal, v. 11, n. 16, p. 39-47, jan./abr., 2010.

EHLING, A.; GRECO, P. Manual de testes. Universidade Federal de Minas Gerais. Escola de Educação Física, Fisioterapia e Terapia Ocupacional. Março, 2005.

FERNANDES, R. Índice de Desenvolvimento da Educação Básica (Ideb). Brasília: Instituto Nacional de Estudos e Pesquisas Educacionais Anísio Teixeira, 2007.

FIN, G.; BARRETO, D. B. M. B. Avaliação motora de crianças com indicadores dificuldades no aprendizado escolar, no município de Fraiburgo, Santa Catarina. Unoesc \& Ciência - ACBS, Joaçaba, v. 1, n. 1, p. 5-12, jan./jun, 2010.

GORLA, J. I.; ARAÚJO, P. F. Avaliação motora em educação física adaptada: teste KTK para deficientes mentais. São Paulo: Phorte, $2007.52 \mathrm{p}$. 
GORLA, J. I.; DUARTE, E.; MONTAGNER, P. C. Avaliação da coordenação motora de escolares da área urbana do Município de Umuarama-PR Brasil. Revista Brasileira de Ciência e Movimento, Brasília, v. 16, n. 2, p. 57-65, 2008.

GRAMANI, M. C. N.; DUARTE, A. L. C. M. O impacto do desempenho das instituições de educação básica na qualidade do ensino superior. Ensaio: Avaliação Política Pública Educação, Rio de Janeiro, v. 19, n. 72, p. 679-702, jul./set., 2011.

GRECO, P. J.; SILVA, S. A.. A metodologia de ensino dos esportes no marco do programa segundo tempo. In: OLIVEIRA, A. B.; PERIM, G. L. (Eds.). Fundamentos pedagógicos para o programa segundo tempo. Porto Alegre: UFRGS, 2008. p.86-136.

GRECO, P. J., SILVA, S. A.; SANTOS, L. R. Organização e Desenvolvimento Pedagógico do Esporte no Programa Segundo Tempo. In: OLIVEIRA, A. A. B.; PERIM, G. L. (Eds.). Fundamentos pedagógicos do programa segundo tempo: da reflexão a prática. Maringá: Eduem, 2009. p.163-206.

KIPHARD, E. J. Insuficiencias de movimiento y de coordinación en la edad de la escuela primaria. Buenos Aires: Editorial Kapelusz. 1976.

KIPHARD, E. J.; SCHILLING, V. F.. Köperkoordinationstest Für Kinder KTK: manual Von Fridhelm Schilling. Weinheim: Beltz Test Gmbh. 1974.

KRÖGER, C.; ROTH, K.. Escola da Bola: Um ABC para iniciantes nos jogos esportivos. São Paulo. 2002.

MEDINA-PAPST, J.; MARQUES, I. Avaliação do desenvolvimento motor de crianças com dificuldades de aprendizagem. Revista Brasileira de Cineantropometria e Desempenho Humano, Florianópolis, v. 12, n. 1, p. 36-42, jan./fev., 2010.

MINAS GERAIS. Conteúdo Básico Comum (CBC). Proposta Curricular: Educação Física, Ensino Fundamental. Secretaria de Estado de Educação de Minas Gerais, 2007a. 
MINAS GERAIS. Conteúdo Básico Comum (CBC). Proposta Curricular: Educação Física, Ensino Médio. Secretaria de Estado de Educação de Minas Gerais, $2007 b$.

NETO, A. S. et al. Relação entre fatores ambientais e habilidades motoras básicas em crianças de 6 e 7 anos. Revista Mackenzie de Educação Física e Esporte, São Paulo, v. 3, n. 3, p. 135-140, 2004.

NETO, F. R. et al. A Importância da avaliação motora em escolares : análise da confiabilidade da Escala de Desenvolvimento Motor. Revista Brasileira de Cineantropometria e Desempenho Humano, Florianópolis, v. 12, n. 6, p. 422-427, nov./dez., 2010.

NETO, F. R. et al. O esquema corporal de crianças com dificuldade de aprendizagem. Revista Semestral da Associação Brasileira de Psicologia Escolar e Educacional, São Paulo, v. 15, n. 1, p. 15-22, jan./jun., 2011.

SILVA, S. A. Bateria de testes para medir a coordenação com bola de crianças e jovens. 2010. 77 f. Tese (Doutorado em Ciências do Movimento Humano)-Escola de Educação Física, Universidade Federal do Rio Grande do Sul, Porto Alegre, 2010.

VALDIVIA, A. B. et al. Prontitud coordinativa: perfiles multivariados en función de la edad, sexo y estatus socio-económico. Revista Portuguesa de Ciências do Desporto, Porto, v. 8, n. 1, p. 34-46, abr., 2008.

Recebido em: 18/04/2012

Revisado em: 05/08/2012

Aprovado em: 09/12/2012

Endereço para correspondência

siomarasilva@cedufop.ufop.br

Siomara Aparecida Silva

Universidade Federal de Ouro Preto

Centro Desportivo da UFOP - CEDUFOP.

Campus Universitario - Morro do Cruzeiro

Bauxita

35400-000 - Ouro Preto, MG - Brasil 